and very dilute sulphuric acid and the mixture was filtered with the aid of a pump in such a manner that both acid and ligroin were drawn through the filter. The acid solution and the ligroin were then separated and the strychnine was recovered from the solution and purified essentially as before. A considerably larger amount was obtained from this portion than from the first. The strychnine obtained was identified by the characteristic reaction with potassium pyrochromate and sulphuric acid, by the intensely bitter taste, by the crystalline form and clouble refraction as seen with the microscope, by the crystals obtained from the chloride with potassium chromate, and by the effect of about is of a milligram upon a frog weighing about two grams. The frog died from the effects of the poison, the symptoms of tetanus being fully developed within ten minutes after the close was administered. The crystals of the chromate were chiefly of the branching forms; there was a little evidence of octahedra but they were not positively identified. The branching forms, however. developed the characteristic violet color when touched with concentrated sulphuric acid.

The total amount of strychnine recovered was estimated to be about two milligrams.

In the trial the chemical evidence was not contested. The defendant was, however, acquitted on other grounds.

Rosi: DULYTECHNK INSTTUT:

DECEMUR 23, 2802

\title{
NOTES ON DETERMINATION OF NICKEL IN STEEL.'
}

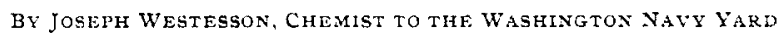

T

$\mathrm{HE}$ desirability of being able to determine nickel in steel

by means of a shorter process than the one, now mostly in use, gave me cause some time ago, when a number of sanples from nickel-steel plates were submitted for analysis to the ordnance laboratory, and when the tedioumess of the said process became particularly apparent, to make some investigations in order to find a more direct method, and I shall beg permission to mention in a few words, what my trials led to.

In the process, above mentioned, the nickel is precipitated

1 Read bytitle at the Baltimore meeting, December 23,1893 . 
by means of hydrogen sulphide in the acetate solution, obtained by separating iron from nickel; the sulphide is filtered off, dried, ignited, dissolved in aqua regia and converted into the double sulphate with ammonia in order to obtain a suit able solution for electrolysis.

It occurred to me, that there ought to be some way, by which the nickel could be electrolytically precipitated directly in the acetate solution, and thus that part of the procedure, embracing the precipitation with hydrogen sulphide, entirely eliminated. If this could be done, it is evident, that there would be quite a saving of time, besides doing away with an unpleasant feature of the method. It became, therefore, my aim to simplify the old method in this peculiar part.

Now, as the presence of chlorides will prevent the electrolysis of nickel, and as the separation of the iron from nickel always had taken place in a chloride solution, I decided to bring my original solution into the shape of sulphates and then proceed on the old lines. After having made a number of more or less successful trials, I finally came to the conclusion, that the following method would answer the purpose very well:

One gram of the sample is dissolved in twenty cc. of sulphuric acid of I.I $6 \mathrm{sp}$. gr., and the solution kept boiling for some time, whereupon five cc. of weak nitric acid is added in small portions. The solution is now evaporated until all the nitric acid is expelled, allowed to cool, water added and heated until clear. Neutralization with carbonate of soda is done in the usual way. but care should be taken not to carry it too close to the point of neutralization. Add water so as to make the bulk 375-400 cc. and precipitate with acetate of soda; filter into a casserole and evaporate the filtrate over a moderate flame; redissolve the ferric precipitate and repeat the precipitation twice, taking care not to make the bulk more than $400 \mathrm{cc}$. each time, and adding each filtrate to the first one. When the filtrates have been evaporated down to $400 \mathrm{cc}$, add ten cc. of concentrated ammonia and boil for a few minutes. The manganese will then settle out, evidently as $\mathrm{H}_{2} \mathrm{O}_{2} \mathrm{MnO}$, and is filtered off, whereupon the filtrate is ready for electrolysis. If the ammonia should be added to the concentrated filtrates, when cold, the manganese 
will not separate out but will interfere considerably with the electrolysis later; it will in that case not only settle out at the anode, but will in fact contaminate the nickel on the cathode, not as loosely settled dioxide but alloyed with the nickel. When, on the other hand, the manganese is precipitated by ammonia in the hot solution, the nickel is to a small extent carried along with the manganese, but I have in all my experiments never found the nickel, lost in that way, amount to more than one to one and a half per cent. of the amount of nickel present in the steel.

How this modification of the old process compares with the original a few results will show.

In a nickel-steel, containing 3.50 per cent. nickel, determined by the old process, the amount found by the modified process in five different analyses was: 3.42 per cent.- $3.5^{1}$ per cent. -3.48 per cent. -3.5 I per cent. -3.43 per cent., and in another steel containing 0.19 per cent., the following amounts were found: 0.18 per cent.-O.I9 per cent.-O.I9 per cent.

The time necessary for analysis of this kind I have found to be about eight hours. Thus, if the analysis is commenced at 8 A.M. the solution will be ready for electrolysis at twelve o'clock, or before; with seven or eight Grove cells in good condition the analysis should be concluded at 4 P.M. I have invariably commenced at noon and left my cylinder over night on four Grove cells, the separation of iron from nickel being completed about four o'clock, or sometimes a little later.

\section{A CORRECTION.}

BY FI. L. PAYNE.

Received January $29,+2894$

IN the Journal of Analytical and Applied Chemistry for September, 1892,6 , No. 9, I' published 'A Method for the Preparation of a Standard Iodine Solution,' and on page 482 I have taken the molecular weight of two atoms of iron as 128 instead of II 2. This singular error which vitiates the entire calculation following it, has been called to my attention by several readers and I desire to correct the mistake and again call attention to the method itself. If any one has been unfortunate 